\title{
A Lower Bound for the Differences of Powers of Linear Operators
}

\author{
J. MALINEN ${ }^{1)}$ O. NEVANLINNA, V. TURUNEN Z. YUAN \\ Department of Mathematics, Helsinki University of Technology, \\ P. O. Box 1100 FIN-02015 HUT, Finland \\ E-mail: Jarmo.Malinen@hut.fi
}

\begin{abstract}
Let $T$ be a bounded linear operator in a Banach space, with $\sigma(T)=\{1\}$. In 1983, Esterle-Berkani' s conjecture was proposed for the decay of differences $(I-T) T^{n}$ as follows: Either $\liminf _{n \rightarrow \infty}(n+1)\left\|(I-T) T^{n}\right\| \geq 1 / e$
\end{abstract}

or $T=I$. We prove this claim and discuss some of its consequences.

Keywords Esterle-Berkani's conjecture, Quasi-nilpotent linear operator, Differences of powers, Decay MR(2000) Subject Classification 47A30, 47D03, 47A10, 30C45

\section{Introduction}

Let $T \in \mathscr{L}(X)$, a bounded linear operator in a (complex) Banach space $X$. The following result by Esterle holds, see [1, Corollary 9.5]:

Proposition 1 Let $T \in \mathscr{L}(X)$ satisfy $\sigma(T)=\{1\}$. If $T \neq I$ then $\liminf _{n \rightarrow \infty}(n+1) \|(I-T)$ $T^{n} \| \geq \frac{1}{96}$.

Berkani improved the lower bound to $1 / 12$, and he conjectured that the best lower bound is $1 / e$, see [2]. That $1 / e$ has a special role in related estimates can also be seen in the following remark by Nevanlinna, see [3, Theorem 4.5.1]:

Proposition 2 Assume that there exists $\left\{\lambda_{j}\right\} \subset \sigma(T)$ such that $\left|\lambda_{j}\right|<1$ and $\left|\lambda_{j}\right| \rightarrow 1$ as $j \rightarrow \infty$. Then $\lim \sup _{n \rightarrow \infty}(n+1)\left\|(I-T) T^{n}\right\| \geq \frac{1}{e}$.

The constant $1 / e$ also appears in the well-known "continuous time" case [4, Theorem 10.3.6].

In this paper, we show that Berkani's and Esterle's conjecture is right in the sense that Proposition 1 holds with $1 / 96$ replaced by $1 / e$. We use a related but more careful analysis that has already been used in [1], involving the univalent functions $g_{n}(z)=z(1-z)^{n}$. Also we give another variant of Proposition 2 without restrictions on $\sigma(T)$.

All of these results were first presented in [5] (Yuan, 2002) with somewhat longer proofs. That $1 / e$ in Proposition 1 is a valid lower bound, is also proved in [6] (Kalton, MontgomerySmith, Oleszkiewicz, and Tomilov, 2002) by quite different means. Both of the existing approaches can be generalized to a larger class of results, but these respective classes are different (and we shall not discuss these generalizations here). An example is given in [6], indicating that the constant $1 / e$ is the best possible. The construction is a modification of an example given in [7] (Lyubich, 2001).

\section{Estimating $\liminf \operatorname{in}_{n \rightarrow \infty}(n+1)\left\|(I-T) T^{n}\right\|$}

Denote $\mathbb{D}(R):=\{z \in \mathbb{C}:|z|<R\}$, and let $g: \mathbb{D}(R) \rightarrow \mathbb{C}$ be an analytic function satisfying $g(0)=0$ and $g^{\prime}(0) \neq 0$. Then there exists a maximal radius $R_{u}, 0<R_{u} \leq R$, such that $g$ is a univalent (i.e. an injective analytic) function on the disk $\mathbb{D}\left(R_{u}\right)$. It is then easy to see that

Received June 25, 2004, Accepted September 15, 2004

1) Corresponding author 
the image of $g\left(\mathbb{D}\left(R_{u}\right)\right)$ contains an open disc, centered at origin. Let $0<c<\infty$ be the largest radius such that $\mathbb{D}(c) \subset g\left(\mathbb{D}\left(R_{u}\right)\right)$. Then there exists an analytic function $f: \mathbb{D}(c) \rightarrow \mathbb{D}\left(R_{u}\right)$ such that

$$
(g \circ f)(z):=g(f(z))=z \quad \text { for all } \quad z \in \mathbb{D}(c) .
$$

We denote the spectral radius of $L \in \mathscr{L}(X)$ by $\rho(L)$. If $\rho(L)=0$, then $L$ is called quasinilpotent. With these notations, we can prove the following proposition:

Proposition 3 Let $g: \mathbb{D}(R) \rightarrow \mathbb{C}$ be an analytic function such that $g(0)=0$ and $g^{\prime}(0) \neq 0$. Let the constants $c$ and $R_{u}$ be as above. Then, for all $0<\eta<1$,

$$
\inf \left\{\|g(L)\|: L \in \mathscr{L}(X), \rho(L)=0,\|L\| \geq R_{u} \eta(1-\eta)^{-1}\right\} \geq \eta c .
$$

Proof The proof is carried out by showing that the set

$$
\left\{L \in \mathscr{L}(X): \rho(L)=0,\|g(L)\|<\eta c,\|L\| \geq R_{u} \eta(1-\eta)^{-1}\right\}
$$

is empty for all $0<\eta<1$. This is achieved by using the Cauchy estimates for the function $f$ defined in (1). Denote the power series representations by $f(z)=\sum_{j>1} a_{j} z^{j}$ and $g(z)=$ $\sum_{j \geq 1} b_{j} z^{j}$. Clearly $f: \mathbb{D}(c) \rightarrow \mathbb{D}\left(R_{u}\right)$ means that $\sup _{|z|<c}|f(z)| \leq R_{u}$, and then the Cauchy estimates give $\left|a_{j}\right| r^{j} \leq R_{u}$ for each $r<c$ and $j \geq 1$. Letting $r \rightarrow c-$, we get that $\left|a_{j}\right| c^{j} \leq R_{u}$ for all $j \geq 1$.

Let $L \in \mathscr{L}(X)$ be an arbitrary quasi-nilpotent operator. Then $g(L)$ is quasi-nilpotent by the spectral mapping theorem, as $g(0)=0$. Similarly $Y:=f(g(L))$ is also quasi-nilpotent. Now let $0<\eta<1$, and assume that $\|g(L)\|<\eta c$. It now follows from the above Cauchy estimates that

$$
\|Y\| \leq \sum_{j \geq 1}\left|a_{j}\right| \cdot\|g(L)\|^{j}<\sum_{j \geq 1}\left|a_{j}\right| c^{j} \cdot \eta^{j} \leq R_{u} \eta(1-\eta)^{-1}
$$

hence $\|Y\|<R_{u} \eta(1-\eta)^{-1}$.

We proceed to show that $Y=L$. Since $Y$ is quasi-nilpotent, $g(Y)$ is well-defined. By the associativity $g(Y)=g[f(g(L))]=g(f[g(L)])=(g \circ f)(g(L))=g(L)$ because $(g \circ f)(z)=z$ for any $z \in \mathbb{D}(c)$. As $g(0)=0$, it follows that $\sigma(g(L))=\{0\} \subset \mathbb{D}(c)$. Using the power series of $g$, we get

$$
\begin{aligned}
0 & =g(Y)-g(L)=\sum_{j \geq 1} b_{j} Y^{j}-\sum_{j \geq 1} b_{j} L^{j} \\
& =(Y-L)\left(b_{1} I+\sum_{j \geq 2} b_{j}\left[Y^{j-1}+Y^{j-2} L+\cdots+L^{j-1}\right]\right) \\
& =(Y-L)\left(b_{1} I+U\right),
\end{aligned}
$$

where $b_{1}=g^{\prime}(0) \neq 0$ and $U:=\sum_{j \geq 2} b_{j}\left[Y^{j-1}+Y^{j-2} L+\cdots+L^{j-1}\right]$.

We know that $Y=f(g(L))$ is quasi-nilpotent, and it is actually a function of $L$. We now consider function $h$ defined in $\mathbb{D}\left(R_{u}\right)$ as follows:

$$
h(z):=\sum_{j \geq 2} b_{j}\left[f(g(z))^{j-1}+f(g(z))^{j-2} z+\cdots+z^{j-1}\right] .
$$

Then $h(z)$ is analytic in $\mathbb{D}\left(R_{u}\right)$ and $h(0)=0$. So $h(L)$ is well defined and $U=h(L)$. Since both $L$ and $Y$ are quasi-nilpotent, we see that $U$ is quasi-nilpotent. Therefore $b_{1} I+U$ is boundedly invertible. This together with (2) implies that $Y=L$. Hence, for any $0<\eta<1$ and any quasi-nilpotent $L \in \mathscr{L}(X),\|g(L)\|<\eta c \Rightarrow\|L\|=\|Y\|<R_{u} \eta(1-\eta)^{-1}$. This proves the claim.

A somewhat analogous result to the previous proposition is [6, Theorem 4.5]. We proceed to study the functions

$$
g_{n}(z):=(1-z)^{n} z \quad \text { for } n \geq 1,
$$

that also made their appearance in Esterle's original argument. We shall make use of the constants $R_{u}^{(n)}$ and $c^{(n)}$ defined as follows: 
1) $R_{u}^{(n)}>0$ is the largest radius of an open disc $\mathbb{D}\left(R_{u}^{(n)}\right)$ such that $g_{n}(z)$ is univalent in $\mathbb{D}\left(R_{u}^{(n)}\right)$;

2) $c^{(n)}>0$ is the largest radius of an open disc $\mathbb{D}\left(c^{(n)}\right)$ such that $\mathbb{D}\left(c^{(n)}\right) \subset g_{n}\left(\mathbb{D}\left(0, R_{u}^{(n)}\right)\right)$.

Because $g_{n}^{\prime}(z)=(1-z)^{n-1}(1-(n+1) z)$ and hence $g_{n}^{\prime}(1 /(n+1))=0$, it follows, by the elementary function theory, that $R_{u}^{(n)} \leq 1 /(n+1)$. The next proposition shows that equality holds here.

Proposition 4 The functions $g_{n}(z)=(1-z)^{n} z$ are univalent in the disc $\mathbb{D}(1 /(n+1))$ for all $n \geq 1$.

Proof Let $z=r e^{i \phi} \in \mathbb{C}$, where $0 \leq r<1 /(n+1)$ and $\phi \in \mathbb{R}$. Now $g_{n}(z)=R(r, \phi) e^{i \Phi(r, \phi)}$, where $r_{\phi}=\sqrt{1-2 r \cos (\phi)+r^{2}}, \Phi(r, \phi)=\phi-n \arcsin \left(r \sin (\phi) / r_{\phi}\right)$ and $R(r, \phi)=r \cdot r_{\phi}^{n}$; note that $\arcsin :[-1,1] \rightarrow[-\pi / 2, \pi / 2]$ is the inverse function of $\sin :[-\pi / 2, \pi / 2] \rightarrow[-1,1]$. Mapping $\phi \mapsto \Phi(r, \phi)$ is injective on $\mathbb{R}$, because, by writing $t=\cos (\phi)$,

$$
\begin{aligned}
\frac{\partial \Phi(r, \phi)}{\partial \phi} & =\left(1-(n+2) r t+(n+1) r^{2}\right)\left(1-2 r t+r^{2}\right)^{-1} \\
& \geq\left(1-(n+2) r+(n+1) r^{2}\right)\left(1-2 r t+r^{2}\right)^{-1} \\
& =(1-r)(1-(n+1) r)\left(1-2 r t+r^{2}\right)^{-1}>0
\end{aligned}
$$

where the last estimate follows as $r<1 /(n+1)$. Notice, furthermore, that $\Phi(r, 2 \pi k)=2 \pi k$ for every $k \in \mathbb{Z}$. Moreover, if $\phi$ is fixed then $\frac{\partial R(r, \phi)}{\partial r}=\frac{\partial \Phi(r, \phi)}{\partial \phi}\left(1-2 r t+r^{2}\right)^{n / 2}>0$. Hence $r \mapsto R(r, \phi)$ is injective on $[0,1 /(n+1))$, and the claim follows.

In other words, we have now proved that $R_{u}^{(n)}=1 /(n+1)$ for all $n \geq 1$. The other sequence of constants can be determined easily.

Proposition 5 The constants $c^{(n)}$ (as introduced earlier) satisfy $c^{(n)}=\frac{1}{n+1}\left(1-\frac{1}{n+1}\right)^{n}$, for all $n \geq 1$.

Proof Clearly, for any fixed $n, c^{(n)}=\inf _{z \in \partial \mathbb{D}\left(R_{u}^{(n)}\right)}\left|g_{n}(z)\right|$. Since $\left|(1-z)^{n} z\right| \geq\left(1-R_{u}^{(n)}\right)^{n} R_{u}^{(n)}$, for all $z$ satisfying $|z|=R_{u}^{(n)}$, we get $c^{(n)} \geq \frac{1}{n+1}\left(1-\frac{1}{n+1}\right)^{n}$ as $R_{u}^{(n)}=1 /(n+1)$ by Proposition 4 . By choosing $z=R_{u}^{(n)}$, we see that even the equality holds.

Now we are prepared to prove our main result. The required improvement of Proposition 1 follows by taking $L=I-T$ in the following theorem:

Theorem 1 Let $L \in \mathscr{L}(X), L \neq 0$, be quasi-nilpotent. Then $\liminf _{n \rightarrow \infty}(n+1)\left\|(I-L)^{n} L\right\|$ $\geq \frac{1}{e}$.

Proof Define the functions $g_{n}$ and the constants $R_{u}^{(n)}, c^{(n)}$ as earlier. Let $0<\eta<1$ be arbitrary. Since by Proposition $4, R_{u}^{(n)} \eta(1-\eta)^{-1}=\frac{1}{n+1} \cdot \eta(1-\eta)^{-1} \rightarrow 0$ as $n \rightarrow \infty$, there exists $N(\eta)<\infty$, such that, for all $n \geq N(\eta)$, we have $\|L\| \geq R_{u}^{(n)} \eta(1-\eta)^{-1}$. By Proposition 3 (with $g=g_{n}$ ) and Proposition 5, we have, for all $n \geq N(\eta)$,

$$
\left\|(I-L)^{n} L\right\| \geq \eta c^{(n)}=\eta \frac{1}{n+1}\left(1-\frac{1}{n+1}\right)^{n+1} .
$$

Since $\lim _{n \rightarrow \infty}\left(1-\frac{1}{n+1}\right)^{n+1}=1 / e$, we get, by letting $n \rightarrow \infty, \liminf _{n \rightarrow \infty}(n+1)\left\|(I-L)^{n} L\right\| \geq$ $\eta / e$. Because $0<\eta<1$ is arbitrary, the claim follows by letting $\eta \rightarrow 1$.

3 Estimating $\lim \sup _{n \rightarrow \infty}(n+1)\left\|(I-T) T^{n}\right\|$

Theorem 2 For any $T \in \mathscr{L}(X)$, either

(i) $\limsup _{n \rightarrow \infty}(n+1)\left\|(I-T) T^{n}\right\| \geq 1 / e$; or,

(ii) $\limsup _{n \rightarrow \infty}(n+1)\left\|(I-T) T^{n}\right\|=0$ holds.

Proof If $\lim _{\sup _{n \rightarrow \infty}}(n+1)\left\|(I-T) T^{n}\right\|=\infty$ or $T=I$, then the claim holds. It remains to consider the case when $\sup _{n \geq 0}(n+1)\left\|(I-T) T^{n}\right\|<\infty$ and $T \neq I$. By [3, Theorem 4.2.2], 
$\sigma(T) \subset \mathbb{D}(1) \cup\{1\}$

If $1 \notin \sigma(T)$, then $\left\|T^{n}\right\| \leq M r^{n}$ for some $0 \leq r<1$, and (ii) follows. If 1 is an accumulation point of $\sigma(T)$, then (i) holds, by Proposition 2. If 1 is an isolated point, then either $\sigma(T)=\{1\}$ or there is a positive distance between 1 and $\sigma(T) \backslash\{1\}$. If $\sigma(T)=\{1\}$, then (i) holds by Theorem 1.

To complete the proof, we can assume dist $(1, \sigma(T) \backslash\{1\})>0$. There exist closed, nonintersecting curves $\Gamma_{1}$ and $\Gamma_{2}$ with the following properties: $\Gamma_{1}$ lies strictly inside the open unit disc $\mathbb{D}(1)$ and it surrounds the set $\sigma(T) \backslash\{1\} ; \Gamma_{2}$ surrounds point 1 . Define the bounded spectral projections $P_{1}$ and $P_{2}$, together with the corresponding closed subspaces $P_{1}:=\frac{1}{2 \pi i} \int_{\Gamma_{1}}(\lambda-T)^{-1} d \lambda$, $P_{2}:=\frac{1}{2 \pi i} \int_{\Gamma_{2}}(\lambda-T)^{-1} d \lambda, X_{1}:=P_{1} X$ and $X_{2}:=P_{2} X$.

Both $X_{1}$ and $X_{2}$ are invariant for $T, X_{1} \cap X_{2}=\{0\}$ and $X=X_{1}+X_{2}$. They inherit their

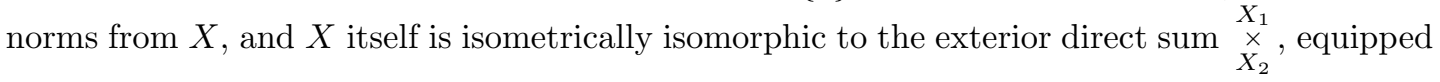
with the norm $\left\|\left[\begin{array}{ll}x_{1} & x_{2}\end{array}\right]^{T}\right\|_{X_{1} \times X_{2}}:=\left\|x_{1}+x_{2}\right\|$ for all $x_{1} \in X_{1}, x_{2} \in X_{2}$. Define the bounded operators $L$ and $M$ by $L:=T \mid X_{1} \in \mathscr{L}\left(X_{1}\right)$ and $M:=T \mid X_{2} \in \mathscr{L}\left(X_{2}\right)$. Then $T$ is isometrically

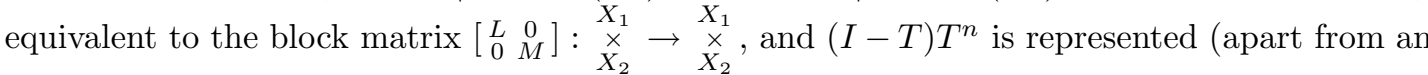
isometric isomorphism) by $\left[\begin{array}{cc}\left(I_{X_{1}}-L\right) L^{n} & X_{2} \\ 0 & \left(I_{X_{2}}-M\right) M^{n}\end{array}\right]$. By the triangle inequality

$$
\begin{aligned}
& \left\|(I-T) T^{n}\right\|=\left\|\left[\begin{array}{cc}
\left(I_{X_{1}}-L\right) L^{n} & 0 \\
0 & \left(I_{X_{2}}-M\right) M^{n}
\end{array}\right]\right\|_{\mathscr{L}\left(X_{1} \times X_{2}\right)} \\
& \geq\left\|\left[\begin{array}{l}
0 \\
0 \\
0\left(I_{X_{2}}-M\right) M^{n}
\end{array}\right]\right\|_{\mathscr{L}\left(X_{1} \times X_{2}\right)}-\left\|\left[\begin{array}{cc}
\left(I_{X_{1}}-L\right) L^{n} & 0 \\
0 & 0
\end{array}\right]\right\|_{\mathscr{L}\left(X_{1} \times X_{2}\right)} \\
& =\left\|\left(I_{X_{2}}-M\right) M^{n}\right\|_{\mathscr{L}\left(X_{2}\right)}-\left\|\left(I_{X_{1}}-L\right) L^{n}\right\|_{\mathscr{L}\left(X_{1}\right)} \text {. }
\end{aligned}
$$

The spectra of $L$ and $M$ satisfy $\sigma(L)=\sigma(T) \backslash\{1\} \subset \mathbb{D}(1)$ and $\sigma(M)=\{1\}$. It follows again immediately that $\lim _{n \rightarrow \infty}(n+1)\left\|\left(I_{X_{1}}-L\right) L^{n}\right\|_{\mathscr{L}\left(X_{1}\right)}=0$. By Theorem 1, $\lim _{\sup _{n \rightarrow \infty}}(n+$ 1) $\left\|\left(I_{X_{2}}-M\right) M^{n}\right\|_{\mathscr{L}\left(X_{2}\right)} \geq 1 / e$. Therefore (4) implies

$$
\limsup _{n \rightarrow \infty}(n+1)\left\|T^{n}(T-1)\right\| \geq \limsup _{n \rightarrow \infty}(n+1)\left\|\left(I_{X_{2}}-M\right) M^{n}\right\|_{\mathscr{L}\left(X_{2}\right)} \geq 1 / e,
$$

and the proof is completed.

The lower bound 1/e in Theorem 2 can be reached, see [3, Example 4.5.2].

\section{References}

[1] Esterle, J.: Quasimultipliers, representations of $H^{\infty}$, and the closed ideal problem for commutative Banach algebras, In Radical Banach algebras and automatic continuity (Long Beach, Calif., 1981), volume 975 of Lecture Notes in Mathematics, 66-162, Springer-Verlag, 1983

[2] Berkani, M.: Inégalités et propriétés spectrales dans les algébres de Banach, Thèse de 3éme cycle, University of Bordeaux I, 1983

[3] Nevanlinna, O.: Convergence of Iterations for Linear Equations, Lectures in Mathematics ETH, BirkhäuserVerlag, Zürich, 1993

[4] Hille, E., Phillips, R. S.: Functional analysis and semi-groups, American Mathematical Society, 1957

[5] Yuan, Z.: On the resolvent and Tauberian conditions for bounded linear operators, Licentiate's Thesis, Helsinki University Of Technology, 2002

[6] Kalton, N., Montgomery-Smith, S., Oleszkiewicz, K., Tomilov, Y.: Power-bounded operators and related norm estimates. J. London Math. Soc. (2), 70(2), 463-478 (2004)

[7] Lyubich, Yu.: The single-point spectrum operators satisfying Ritt's resolvent condition. Studia Mathematica, 145, 135-142, (2001) 